\title{
Modeling and Predicting Future Trajectories of Moving Objects in a Constrained Network
}

\author{
Jidong Chen $\dagger \quad$ Xiaofeng Meng $† \quad$ Yanyan Guo $†$ Stéphane Grumbach $\ddagger \quad$ Hui Sun $\dagger$ \\ $\dagger$ Information School, Renmin University of China, Beijing, China \\ \{chenjd, xfmeng, guoyy, hsun\}@ ruc.edu.cn \\ †CNRS, LIAMA, Beijing, China \\ grumbach@liama.ia.ac.cn
}

\begin{abstract}
Advances in wireless sensor networks and positioning technologies enable traffic management (e.g. routing traffic) that uses real-time data monitored by GPS-enabled cars. Location management has become an enabling technology in such application. The location modeling and trajectory prediction of moving objects are the fundamental components of location management in mobile locationaware applications. In this paper, we model the road network and moving objects in a graph of cellular automata (GCA), which makes full use of the constraints of the network and the stochastic behavior of the traffic. A simulation-based method based on graphs of cellular automata is proposed to predict future trajectories. Our technique strongly differs from the linear prediction method, which has low prediction accuracy and requires frequent updates when applied to real traffic with velocity changes. The experiments, carried on two different datasets, show that the simulation-based prediction method provides higher accuracy than the linear prediction method.
\end{abstract}

\section{Introduction}

The continued advances in wireless sensor networks and position technologies enable traffic management and location-based services that track continuously changing positions of moving objects. For example, moving cars on a road network can be monitored and their locations are sampled by sensors or GPS periodically, then sent to the server and stored in a database. According to the real-time locations and predicted future trajectories of cars, we can forecast traffic jams and route the traffic intelligently. Timely location information is becoming one of the key features in these applications. In this paper, we focus on the the location modeling and future trajectory prediction of mov- ing objects, which are the foundations for efficient location management in mobile location-aware applications.

Many models and algorithms have been proposed to handle the continuously changing positions of moving objects. Wolfson et al. in [16, 21] firstly proposed a Moving Objects Spatio-Temporal (MOST) model, which represents the location as a dynamic attribute. Later, the model based on linear constrain [17], abstract data types [9] and SpaceTime Grid Storage [4] for moving objects have been proposed. However, in most real life applications, objects move within constrained networks, especially the transportation networks (e.g., vehicles move on road networks). These works ignore the interaction between moving objects and the underlying transportation networks.

In fact, the interaction is very important to manage network-constrained moving objects. For example, in the location tracking, the road-network representation of moving objects can be exploited to reduce the number of updates from moving objects to the database server [5]. For indexing moving objects in road networks, the temporal aspect can be distinguished and related to the road network to save considerable index storage space $[2,8]$ since the spatial property of objects' movement is already captured by the network. In addition, using the network constraints, the query processing can also be improved [10, 15].

More recently, the models connecting moving objects with the road network representation have been proposed $[7,13,14,20]$. Most of them represent road networks as graphs and moving objects as moving graph points with their speed in order to capture objects' movement. However, the models assume linear movement and can not reflect the real movement feature of moving objects in a road network where objects frequently change their velocity. This limits their applicability in a majority of real applications.

In this paper, we propose a new graph of cellular automata (GCA) model to integrate the traffic movement fea- 
tures into the model of moving objects and the underlying road network. The GCA model exploits the stochastic behavior of the real traffic by the cellular automaton which is used in the traffic simulation [12]. It also combines the road network model with the real movement of objects and therefore improves the efficiency of managing network-constrained moving objects.

Considering the new feature of the GCA model, it can be efficiently used to simulate future trajectories of moving objects, where objects' movement follows traffic rules. We further propose a simulation-based prediction method based on the GCA model. Since the GCA exploits features of traffic systems, the method can predict future trajectories of moving objects in road network more accurately than the linear prediction method widely used in the predictive indexing and query processing.

The framework built on the GCA model and simulationbased prediction forms the foundation of the efficient storage and management of network-constrained moving objects. Specifically, it is capable of reducing the number of updates in tracking and indexing and supporting the predictive queries on moving objects in a road network.

In summary, this paper makes the following contributions:

1. We present the graphs of cellular automata (GCA) model to integrate the trajectory representation of moving objects and the transportation network with intrinsic movement features in the real traffic.

2. Based on the GCA model, we propose a simulationbased prediction (SP) method, which improves the accuracy of predicting future trajectories of objects moving in a traffic network.

3. The experiments show that the simulation-based prediction method obtains higher accuracy than the linear prediction method widely used.

The rest of the paper is organized as follows. Section 2 surveys related work. In Section 3, the graph of cellular automata model is introduced to model the road network and movement of objects. Section 4 presents the simulationbased prediction method. Section 5 contains experimental evaluation. We conclude in Section 6.

\section{Related Work}

The modeling of moving objects attracts a lot of research interests. Wolfson et al. in [16, 21] firstly proposed a Moving Objects Spatio-Temporal (MOST) model which is capable of tracking not only the current, but also the near future position of moving objects. Su et al. in [17] presented a data model for moving objects based on linear constraint databases. Chon et al. in [4] proposed a Space-Time Grid
Storage model for moving objects. In [9], Güting et al. presented a data model and data structures for moving objects based on abstract data types. However, nearly none of these works have treated the interaction between moving objects and the underlying transportation networks in any way.

In 2001, Vazirgiannis and Wolfson [20] first introduced a model for moving objects on road networks, which connects the moving object's trajectory model with the road network representation. In the model, the road network is represented by an electronic map and the trajectory of a moving object is constructed by the map and its destination. In [14], the authors presented a computational data model for network constrained moving objects in which the road network has two representations namely a two-dimensional representation and a graph representation to obtain both expressiveness and efficient support for queries. In this model, the moving objects treated as query points are represented by graph points located on segments or edges. Ding et al. [7] proposed a MOD model, based on dynamic transportation networks. They model transportation networks as dynamic graphs and moving objects as moving graph points. In addition, Papadias et al. in [13] presented a framework to support spatial network databases. However, these models capture movement information of objects only by their speed and assume the linear movement, which limit applicability in a majority of real applications.

Prediction methods for future trajectories of moving objects play an important role in indexing and querying current and anticipated future positions. Most existing prediction methods, used in the indexing and querying, assume linear movement, which cannot reflect the real movement. Aggarwal et al [1] introduced a non-linear model that uses quadratic predictive function, Tao et al [18] proposed a prediction method based on recursive motion functions for objects with unknown motion patterns, and Cai et al [6] used Chebyshev polynomials to represent and index spatio-temporal trajectories. In [19], Tao et al developed Venn sampling (VS), a novel estimation method optimized for a set of pivot queries that reflect the distribution of actual ones. These prediction methods improve the precision in predicting the location of each object, but they ignore the correlation of adjacent objects when they move in traffic networks, and thus may not reflect the realistic traffic scenario.

Despite the wide use of traffic simulation rules in transportation GIS domain [12,3], their integration to a database model for objects in constrained networks has never been done before.

\section{Graphs of Cellular Automata Model}

We model a road network with a graph of cellular automata (GCA), where the nodes of the graph represent road intersections and the edges represent road segments with 


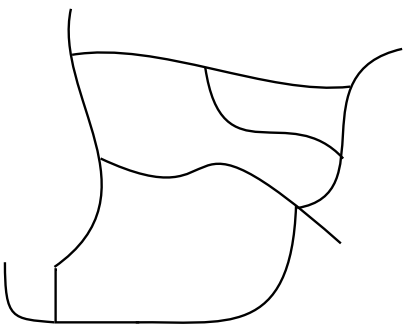

(a) A Road Network

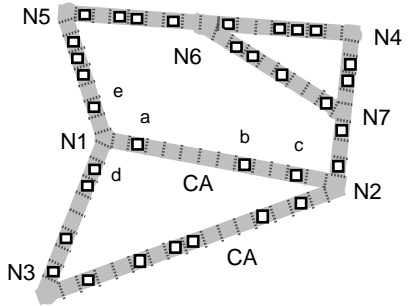

(b) An instance of GCA
Figure 1: An example of a road network and its GCA model

no intersections. Different from the general graph model, each edge in the GCA consists of a cellular automaton (CA), which is represented, in a discrete mode, as a finite sequence of cells. Each cell corresponds in practice to some road segment of about $7.5 \mathrm{~m}$.

Figure 1 shows an example of a road network and its GCA model. Each node has a label which represents an intersection of the road network. The wide lines represent edges and each edge treated as one CA connects many cells.

The CA model was used in this context by [12]. We first recall the definition of cellular automaton.

Definition 1 A cellular automaton consists of a finite oriented sequence of cells. In a configuration, each cell is either empty or contains a symbol. During a transition, symbols can move forward to subsequent cells, symbols can leave the CA and new symbols can enter the CA.

An example of cellular automaton corresponding to edge $\left(N_{1}, N_{2}\right)$ in Figure 1(b) with a transition between two configurations is given in Figure 2. We now formally define a graph of cellular automata.

Definition 2 The structure of a GCA is a directed weighted graph $G=(V, E, l)$ where $V$ is a set of vertices (i.e., nodes), $E$ is a set of edges and $l: E \rightarrow \mathbb{N}$ is a function which associates to each edge the number of cells of the corresponding cellular automaton.

We assume a countably infinite alphabet $\Omega$ : $\{\alpha, \beta, \gamma, \cdots\}$, denoting moving objects' names. Let $C$ be the set of cells of a GCA.

A configuration or an instance of a GCA, is a mapping from the cells of the GCA to constants in $\Omega$ together with a given velocity. Intuitively, the velocity is the number of cells an object can traverse during a time unit.

Definition 3 An instance I of a GCA is defined by two functions:

$$
\begin{aligned}
& \mu: C \rightarrow \Omega \bigcup\{\varepsilon\} \text { (1-1 mapping) } \\
& v: \Omega \rightarrow \mathbb{N} .
\end{aligned}
$$

A moving object is represented as a symbol attached to the cell in the GCA and it can move several cells ahead at each time unit. Figure 1(b) is an instance of the GCA corresponding to the road network of Figure 1(a). In Figure 1(b), moving objects are denoted by squares. A moving object lies on exactly one cell of the edge and its location can be obtained by computing the number of cells relative to the start node. For instance, object $\alpha$ lies on the edge $\left(N_{1}, N_{2}\right)$ and there are two cells away from $N_{1}$ along the edge. Therefore, its position can be expressed by $\left(N_{1}, N_{2}, 2\right)$.

The motion of an object is represented as some (time, location) information. Representing such information of a moving object as a trajectory is a typical approach [20]. In the GCA model, the trajectory of a moving object can be divided two types: the in-edge trajectory for the object's movement in one edge (CA) and the global trajectory for the object that may move cross several edges (CAs) during its movement. The in-edge trajectory of an object is a polyline in two-dimensional space (one-dimensional relative distance, plus time), which can be defined as follows:

Definition 4 The in-edge trajectory of a moving object in a $C A$ of length $L$ is a piece-wise function $f: T \rightarrow \mathbb{N}$, represented as a sequence of points $\left(t_{1}, l_{1}\right),\left(t_{2}, l_{2}\right), \ldots$, $\left(t_{n}, l_{n}\right)\left(t_{1}<t_{2}<\ldots<t_{n}, l_{1}<l_{2}<\ldots<l_{n} \leq L\right)$.

When an object moves across multiple edges, its global trajectory is defined as functions mapping the time to the edge and relative distance.

Definition 5 The global trajectory of a moving object in different $C A s$ is a piece-wise function $f$ : $T \rightarrow(E, \mathbb{N})$, represented as a sequence of points $\left(t_{1}, e_{1}, l_{1}\right), \ldots,\left(t_{i}, e_{j}, l_{k}\right), \ldots,\left(t_{z}, e_{m}, l_{n}\right)\left(t_{1}<t_{2}<\right.$ $\left.\ldots<t_{z}\right)$.

In the sequel, we will be interested by deterministic paths in the GCA i.e., path with source nodes of out degree 1 . The successive CAs in a deterministic path can be then seen as a unique $\mathrm{CA}$.

Let $i$ be an object moving along an edge. Let $v(i)$ be its velocity, $x(i)$ its position, $g a p(i)$ the number of empty cells ahead (forward gap), and $P_{d}(i)$ a randomized slowdown rate which specifies the probability it slows down. We assume that $V_{\max }$ is the maximum velocity of moving objects. The position and velocity of each object might change at each transition as shown definition 6 adapted from [12].

Definition 6 At each transition of the GCA, each object changes velocity and position in a $C A$ of length $L$ according to the rules below:
1. if $v(i)<V_{\max }$ and $v(i)<\operatorname{gap}(i)$ then $v(i) \leftarrow v(i)+1$
2. if $v(i)>\operatorname{gap}(i)$ then $v(i) \leftarrow \operatorname{gap}(i)$ 


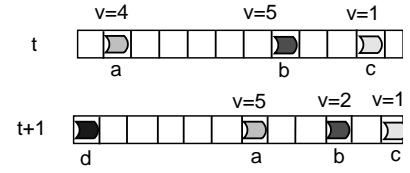

Figure 2: Transition of the GCA

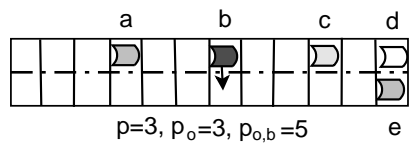

Figure 3: An example of changing lane in transition of the two lane GCA

3. if $v(i)>0$ and $\operatorname{rand}()<P_{d}(i)$ then $v(i) \leftarrow v(i)-1$

4. if $(x(i)+v(i)) \leq L$ then $x(i) \leftarrow x(i)+v(i)$

The first rule represents linear acceleration until the object reaches the maximum speed $V_{\max }$. The second rule ensures that if there is another object in front of the current object, it will slow down in order to avoid collision. In the third rule, the $P_{d}(i)$ models erratic movement behavior. Finally, the new position of object $i$ is given by the fourth rule as the sum of the previous position with the new velocity if it is in the CA. Note that it is easy to extend the definition of transition to deterministic paths. Because of deterministic path, the objects move to a new position in a subsequent CA. Figure 2 shows the simulated movement of objects on a cellular automaton of the GCA in two consecutive timestamps. We can see that at time $t$, the speed of the object $a$ is smaller than the gap (i.e. the number of cells between the object $a$ and $b$ ). On the other hand, the object $b$ will reduce its speed to the size of the gap. According to the fourth rule, the objects move to the corresponding positions based on their speeds at time $t+1$.

However, objects in real traffic have different desired speed. With the transitions of the GCA of one lane CA mentioned above, it can be found that slow objects being followed by faster ones, and the average speed reduced to the free-flow speed of the slowest object [11]. In view of this, we extend the one lane GCA to two lane GCA in which a CA consists of two parallel single lane. Therefore, each cell in two lane GCA is composed of two parallel single lane and each lane may contain one symbol namely a moving object. The function $\mu$ in a GCA instance $I$ will change to the 1-2 mapping accordingly.

For the transition of GCA with one lane, we extend it to the two lane by attaching an additional rule that models the changing of lanes of the object. Suppose the objects move only sideways, the transition of GCA happens on both lanes according to the previous four rules and then the exchange of objects between two lanes is checked according to the additional conditions for changing lane as follows:

\section{5. object $i$ changes lane with probability $P_{c}$ if}

$$
\operatorname{gap}(i)<p, \operatorname{gap}_{o}(i)>p_{1}, \text { and } \operatorname{gap}_{o, b}(i)>p_{o, b}
$$

where $\operatorname{gap}(i)$ is the number of empty cells ahead in the same lane, $\operatorname{gap}_{o}(i)$ is the forward gap on the other lane, $\operatorname{gap}_{o, b}(i)$ is the backward gap on the other lane, $p, p_{o}$ and $p_{o, b}$ are the parameters which decide how far the object looks ahead on the current lane, ahead on the other lane, and back on the other lane, respectively.

In fact, the changing lane rule is based on the following observation: the car looks ahead if some car is in its way; the car looks on the other lane if it is any better there; the car looks back on the other lane if it would get in other cars way. Generally, in the above rule, both $p$ and $p_{o}$ are essentially proportional to the velocity, whereas looking back depends mostly on the expected velocity of other objects, not on one's own. An example of invoking the rule of changing lane with $p=v+1, p_{o}=p, p_{o, b}=v_{\max }, P_{c}=1$ is given in Figure 3. The object $b$ with $p=3, p_{o}=3, p_{o, b}=5$ changes to the other lane in the GCA due to satisfying the fifth rule mentioned above.

\section{Trajectory Prediction}

In the management of moving objects, the trajectory prediction method is usually used to improve the performance of the location update strategy and to support the predictive index and queries. In this part, we first review some linear prediction methods and analyze their problem in handling moving objects in constrained networks, and finally present our simulation-based prediction method.

\subsection{The Linear Prediction (LP)}

Most current index and query processing approaches use the linear prediction method for its simplicity and capability of approximating any curve of free movement by piecewise linear segments. Suppose the trajectory function for an object between time $t_{0}$ and $t_{1}$ is

$$
\vec{X}_{t}=\vec{X}_{t_{0}}+\vec{V}\left(t-t_{0}\right) \quad\left(t_{0} \leq t \leq t_{1}\right)
$$

where $\vec{X}_{t_{0}}$ denotes the position of the object at time $t_{0}$ and $\vec{V}$ denotes the velocity of the object, which is assumed to remain fixed between $t_{0}$ to $t_{1}$.

General LP The general linear prediction method uses the object's current position $\vec{X}_{t_{0}}$ and current velocity $\vec{V}$ to predict its position in the near future. When the prediction is deemed inaccurate, that is, its deviation from the actual position is beyond a predefined threshold, we revise our prediction by resetting $\vec{X}_{t_{0}}$ and $\vec{V}$. In situations where object's velocity remains largely constant, this method enables us to 


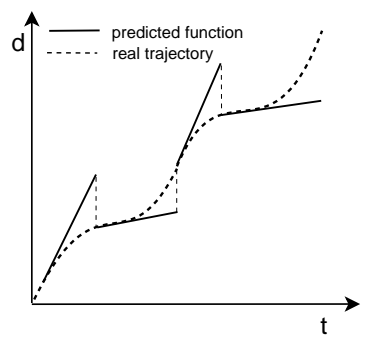

(a) General Linear Prediction

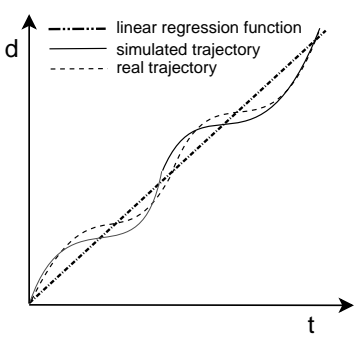

(b) Simulation-based Prediction
Figure 4: Linear Prediction VS. Simulation-based Prediction

make future prediction with high precision. However, when objects move with changing velocity, their trajectory functions have to be revised frequently.

Road Segment Based LP If objects move in a constrained environment such as a transportation network, we can use the road segments of the network to help model the object's movement. In other words, we assume objects move at constant speed along a road segment, that is, their trajectory functions will not change until they move out of a road segment. When an object enters a new road segment, we reset the velocity $\vec{V}$ in its trajectory function. The frequency of revising the trajectory function depends on the average length of the road segments.

Route Based LP If objects have regular and known routes in the transportation network (e.g., one takes the same route from home to work), we can use the routes instead of the road segments to reduce the number of updates needed to maintain the objects' position. If the route is predicted incorrectly, we simply make an additional update.

However, any real traffic system has a stochastic, dynamic and fuzzy nature. The accuracy of linear prediction methods mentioned above is inadequate because linear methods can hardly reflect the movement of objects constrained by road networks. For example, in urban road networks, because of traffic conditions, a vehicle may travel at a constant speed, decelerate to stop, wait, accelerate and travel again at a constant speed. Vehicles may often repeat the above movement in modern urban road networks.

We use Figure 4 to demonstrate the inadequacy of the linear prediction method for real road networks. Figure 4(a) shows the predicted (linear) trajectory and the actual trajectory of an object. We can see that each time the change of the object's velocity is above a certain threshold, an update is triggered and the trajectory is revised by a new velocity vector. The frequent changes of the object's velocity will incur repeated update and prediction.

\subsection{The Simulation-based Prediction (SP)}

Considering the simulation feature of the GCA model, we use GCAs not only to model road networks, but also to simulate future trajectories of moving objects by the transitions of GCAs, where objects' movement follows traffic rules. Based on the GCA, a Simulation-based Prediction $(S P)$ method to anticipate future trajectories of moving objects is proposed. The SP method treats the object's simulated results as its predicted positions to obtain its future in-edge trajectory. To refine the accuracy, based on different assumptions on the traffic conditions we simulate two future trajectories in discrete points for each object on its edge. Then, by linear regression and translating, the trajectory bounds that contain all possible future positions of a moving object on that edge can be obtained. When the object moves to another edge in the GCA or the predicted position exceeds its actual position above the predefined accuracy, another simulation and regression will be executed to predict new future trajectory bounds. The process of the simulation-based prediction can be seen in Figure 5.

Most existing work uses the CA model for traffic flow simulation in which the parameter $P_{d}(i)$ is treated as a random variable to reflect the stochastic, dynamic nature of traffic system. However, we extend this model for predicting the future trajectories of objects by setting $P_{d}(i)$ to values that model different traffic conditions. For example, laminar traffic can be simulated with $P_{d}(i)$ set to 0 or a small value, and the congestion can be simulated with a larger $P_{d}(i)$. By giving $P_{d}(i)$ two values, we can derive two future trajectories, which describe, respectively, the fastest and slowest movements of objects as showed in Figure 5(a). In other words, the object's future locations are most probably bounded by these two trajectories. The value of $P_{d}(i)$ can be obtained by the experiences or by sampling from the given dataset. Our experiments show one of methods to choose the value of $P_{d}(i)$. It is proved that 0 and 0.1 are realistic values of $P_{d}(i)$ in our cases.

For getting the future trajectory function of an object from the simulated discrete points, we need to regress the discrete positions. We find that in most cases the linear regression (as shown in Figure 4b) fits the prediction well and at low cost. The OLSE (Ordinary Least Square Estimation) method, for example, can be calculated efficiently with low data storage cost. Let the discrete simulated points be $\left(t_{1}, d_{1}\right), \ldots,\left(t_{i}, d_{i}\right), \ldots,\left(t_{n}, d_{n}\right)$, where $d_{i}(i \in[1, n])$ denotes the relative distance in an edge and the average value be $\bar{t}$ and $\bar{d}$. After regression, the trajectory function of the moving object is:

$$
D(t)=\hat{\beta}_{0}+\hat{\beta}_{1} \cdot t
$$

where $\hat{\beta}_{o}$ and $\hat{\beta}_{1}$ are given by:

$$
\hat{\beta}_{0}=\bar{d}-\hat{\beta}_{1} \cdot \bar{t}
$$




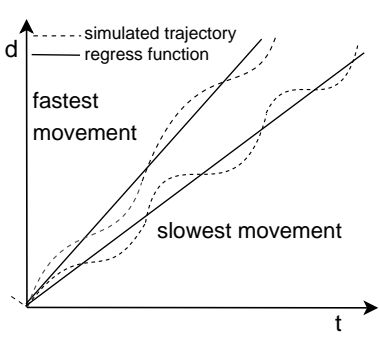

(a) Simulated trajectories (b) Two predicted bounds

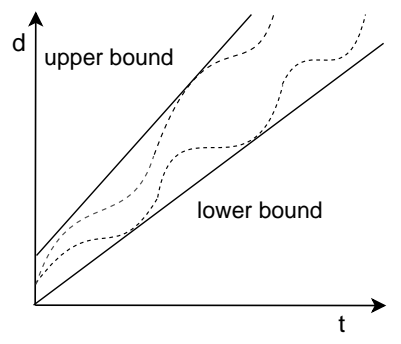

Figure 5: Two Predicted Bounds of Future Trajectories

$$
\hat{\beta}_{1}=\frac{\sum_{i=1}^{n} t_{i} d_{i}-n \bar{t} \cdot \bar{d}}{\sum_{i=1}^{n} t_{i}^{2}-n(\bar{t})^{2}}
$$

In Figure 5(a), the dashed curves show two future trajectories, which are the slowest and the fastest movements simulated by using different $P_{d}$. Applying the OLSE algorithm to the two trajectories generates two linear functions, which are shown in solid lines.

$$
\begin{array}{ll}
\text { fastTrj: } & D(t)=\alpha_{f} \cdot t+\gamma_{f} \\
\text { slowTrj : } & D(t)=\alpha_{s} \cdot t+\gamma_{s}
\end{array}
$$

Finally, in order to find the bounds of the area that contains all estimated future positions, we translate the two regression lines, until all estimated future positions fall within. More specifically, we translate the upper line (fastest movement) upwards until it touches the point with the max residual (denoting $\epsilon_{1}$ the distance translated upward), and similarly, we translate the lower line (slowest movement) downwards (denoting $\epsilon_{2}$ the distance translated downward). This minimizes the loss of information and errors brought by the OLSE algorithm.

We now define the two bound lines as the upper bound and lower bound of objects' future trajectory.

Definition 7 The upper bound of an object trajectory upperBound is the upper bound line of its fastest future trajectory, and the lower bound lowerBound is the lower bound line of its slowest future trajectory. They are linear functions of the following form:

$$
\begin{array}{ll}
\text { upperBound : } & D(t)=\alpha_{f} \cdot t+\lambda_{f} \\
\text { lowerBound : } & D(t)=\alpha_{s} \cdot t+\lambda_{s}
\end{array}
$$

where $\lambda_{f}=\gamma_{f}+\epsilon_{1}, \lambda_{s}=\gamma_{s}-\epsilon_{2}$.

The two bound lines are shown in Figure 5(b). we can treat the two predicted lines as the bounds of the possible future positions of one object. The predicted trajectory bounds can be used in the predictive index structure and query processing in road network to reduce the index updates and filter unnecessary query results to improve the

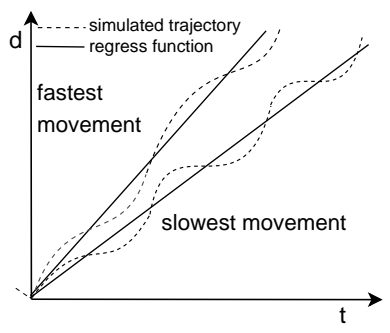

(a) Simulated trajectories

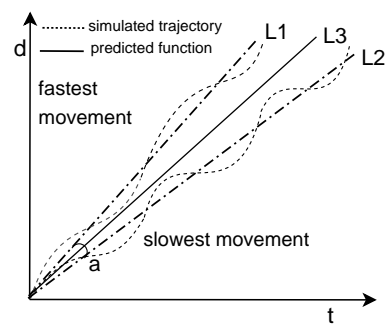

(b) Single predicted function
Figure 6: Singe Predicted Future Trajectory

performance of predictive query. For example, given a predictive range query with the specified region $R$ during time interval $\left[t_{1}, t_{2}\right]$ in the future, we can filter the objects in the result during the pre-process phase if the area between their upper and lower trajectory bounds can not intersect the $R$ during $\left[t_{1}, t_{2}\right]$.

However, for other applications such as the tracking of moving objects, a single predicted function is needed to obtain the specific future positions of the object. For example, to lower update frequency from moving objects to server database, a general principle for location update policies is as follows: the moving objects equipped by GPS receiver do not report their locations to the server unless their actual positions exceed the predicted positions to a certain threshold. Their predicted positions need to be computed by a single predicted function. In this case, we can also adapt the SP method to obtain a compact and simple linear prediction function. The process can be seen in Figure 6. After regressing the two simulated future trajectories to two linear function denoting $L_{1}$ and $L_{2}$, we compute the middle straight line $L_{3}$, the bisector of the angle $a$ between $L_{1}$ and $L_{2}$ as the final predicted function $L(t)$.

Although the predicted function obtained by the SP method is a simple linear function, it is different from the linear prediction in that the SP method not only considers the speed and direction of each moving object, but also takes correlation of objects as well as the stochastic behavior of the traffic into account. The experimental results also show it is a more accurate and effective prediction approach.

As the prediction of in-edge trajectory only use the GCA to simulate the movement of objects in an edge, we have to consider the cases when objects move across the nodes in order to make the global trajectory prediction. If the out degree of a node in the GCA is one, the behavior of the object in the adjacent edge is the same. However, if the out degree of the node is bigger than one, we can not trace the objects cross the different edges. In this case, we could use the probability of objects changing the edges according to the historical data. In this paper, we only predict the in-edge trajectory of the object moving in one edge of the 
GCA. When the object moves to another edge or its prediction accuracy of the future positions cannot arrive the given accuracy requirement, we issue another prediction based on the current traffic conditions.

\section{Experimental Evaluation}

We evaluate the simulation-based prediction method by comparing it with the general linear prediction method. Using two datasets (generated by the CA simulator and by the Brinkhoff's Network-based Generator [3]), we measure their prediction accuracy when applied to predict the near anticipated future positions in the real map network. We also study the effect of the choice of different values of the parameter $P_{d}$ on the simulation-base prediction.

\section{Datasets}

We use two datasets for our experiments. The first is generated by the CA simulator, and the second by the Brinkhoff's Network-based Generator [3]. We use the CA traffic simulator to generate a given number of objects in a uniform network of size $10000 \times 10000$ consisting of 500 edges. Each object has its route and is initially placed at a random position on its route. The initial velocities of the objects follow a uniform random distribution in the range $[0,30]$. The location and velocity of every object is updated at each time-stamp.

The Brinkhoff's Network-based Generator has been used as a popular benchmark in the related work of the MOD. The generator takes a map of a real road network as input (our experiment is based on the map of Oldenburg including 7035 edges). The positions of the objects are given in two dimensional $\mathrm{X}-\mathrm{Y}$ coordinates. We transform them to the form of (edgeid, pos), where edgeid denotes the edge identifier and pos denotes relative position on the edge. The generator places a given number of objects at random positions on the road network, and updates their locations at each time-stamp. Each object has its own destination, and it moves toward its destination along a given route.

\section{Prediction Accuracy and Cost}

We compare the precision of the SP method with the LP method. We measure the prediction accuracy by "average error" but with different threshold. The threshold represents the maximum deviation between the predicted locations of a moving object and its real locations allowed in the prediction. That means when the deviation exceeds the threshold, we make another prediction. From Figure 8, we observe that average error will increase when threshold increases. This is because the larger the threshold is, the larger the deviation becomes, which leads to the more errors. This is tenable in both the LP and SP method. However, the SP method predicts more accurately than the LP method with any threshold.

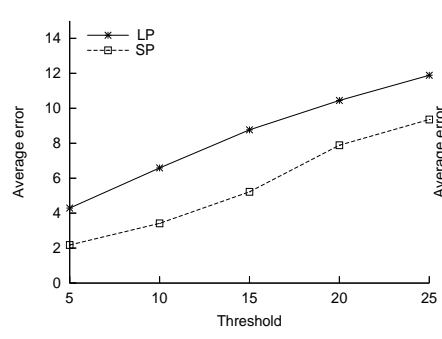

(a) Brinkhoff

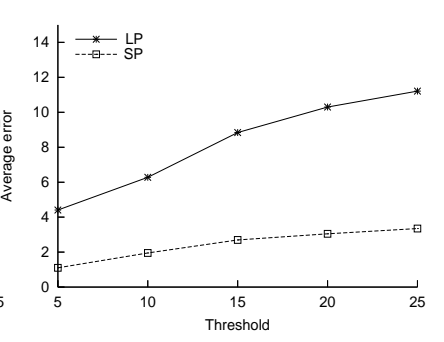

(b) $\mathrm{CA}$
Figure 7: Prediction Accuracy with Different Threshold

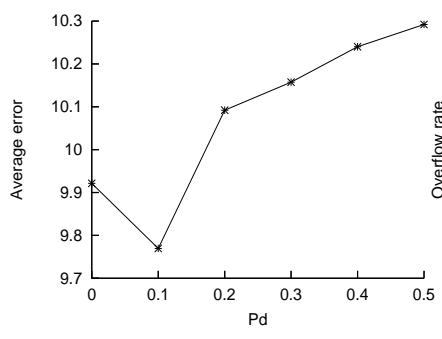

(a) Average Error

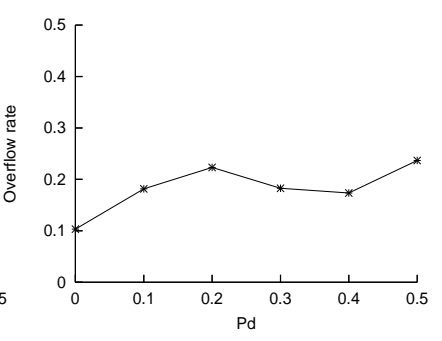

(b) Overflow Rate
Figure 8: Prediction Accuracy with Different $P_{d}$

The time complexity of the simulation-based prediction depends on many factors. We compute the average CPU time when simulating and predicting the movement of one object along the edge with length 1000 in different dataset sizes. The results show that the average cost of one simulation-based prediction is about $0.25 \mathrm{~ms}$. This is quite acceptable.

\section{The Slowdown Rate $P_{d}$}

The CA simulation has an important effect on the accuracy of the simulation-based prediction. We study the effect of the choice of different $P_{d}$, which determines the two predicted trajectories corresponding to the fastest and slowest movement. We test on the Brinkhoff dataset with different data size and use $P_{d}$ from 0 to 0.5 and measure the average prediction accuracy by "average error" and "overflow rate". The average error is the average absolute error between the predicted and actual positions, and the overflow rate represents the probability of predicted positions exceeding the actual positions. The purpose of this metric is to find the closest two trajectories binding the actual one as future trajectories. In this way, we can choose the $P_{d}$ both with lower average error and overflow rate. Figure 8 shows the prediction accuracy of the SP method with different slowdown rates. We can see that when $P_{d}$ is set to 0 and 0.1 , both the average error and overflow rate are lower than others. Therefore, we use the value 0 and 0.1 as slowdown rates for the fastest movement bound and the slowest movement bound to obtain better prediction results. 


\section{Conclusion}

Managing moving objects in a constrained network is a challenging task as well as of great practical importance in mobile location-aware applications. It is necessary to represent and predict the future trajectories of moving objects more accurate. In this paper, we first combine road network representation and the movement model of objects in a traffic network to introduce a new model - GCA for networkconstrained moving objects. And then we propose a prediction method, based on the GCA, which predicts with a great accuracy the future trajectories of moving objects. The accuracy results from the fact that the GCA model exploits the constraints of the network and models the stochastic aspect of urban traffic. Our experimental results performed on two datasets show that the prediction accuracy of our simulation-based prediction is higher than the linear prediction used in the predictive indexing and query processing.

\section{Acknowledgment}

This research was partially supported by the grants from the Natural Science Foundation of China under grant number 60573091, 60273018; the Key Project of Ministry of Education of China under Grant No.03044; Program for New Century Excellent Talents in University (NCET); Program for Creative PhD Thesis in University and performed in the framework of a joint project with INRIA. The authors would like to thank Zhen Xiao, Benzhao Li from Information school, Renmin University of China for the assistance of experimental evaluation and Karine Zeitouni from PRISM, Versailles Saint-Quentin University in France for many helpful advice and assistance.

\section{References}

[1] C. Aggarwal, D. Agrawal. On Nearest Neighbor Indexing of Nonlinear Trajectories. In PODS, 2003, 252-259.

[2] V. T. Almeida, R. H. Güting. Indexing the Trajectories of Moving Objects in Networks (Extended Abstract). In SSDBM, 2004, 115-118.

[3] T. Brinkhof. A framework for generating networkbased moving objects. In GeoInformatica, 6(2), 2002, 153-180.

[4] H. D. Chon, D. Agrawal, A. E. Abbadi. Using SpaceTime Grid for Efficient Management of Moving Objects. In MobiDE 2001, 59-65.

[5] A. Civilis, C. S. Jensen, S. Pakalnis. Techniques for Efficient Road-Network-Based Tracking of Moving Objects. In IEEE Trans. Knowl. Data Eng. 17(5): 698-712 (2005).

[6] Y. Cai, N. Raymond. Indexing spatiotemporal trajectories with chebyshev polynomials. In SIGMOD, 2004, 599-610.
[7] Z. Ding, R. H. Güting. Managing Moving Objects on Dynamic Transportation Networks. In SSDBM, 2004, 287-296.

[8] E. Frentzos. Indexing objects moving on Fixed networks. In SSTD, 2003, 289-305.

[9] R. H. Güting, M. H. Böhlen, M. Erwig, C. S. Jensen, N. A. Lorentzos, M. Schneider, M. Vazirgiannis. A Foundation for Representing and Querying Moving Objects. In TODS 25(1), 1-42(2000).

[10] M. Kolahdouzan, C. Shahabi. Voronoi-Based K Nearest Neighbor Search for Spatial Network Databases. In VLDB 2004, 840-851.

[11] T. Nagatani. Bunching of cars in asymmetric exclusion models for freeway traffic, Phys. Rev. E 51(N2), 922 (1995).

[12] K. Nagel, M. Schreckenberg. A cellular automaton model for freeway traffic. Journal Physique I 2, 1992, 2221-2229.

[13] D. Papadias, J. Zhang, N. Mamoulis, Y. Tao. Query Processing in Spatial Network Databases. In VLDB, 2003, 790-801.

[14] L. Speicys, C. S. Jensen, A. Kligys. Computational Data Modeling for network-Constrained Moving Objects. In ACM-GIS, 2003, 118-125.

[15] C. Shababi, M.R. Kolahdouzan, M. Sharifzadeh. A Road Network Embedding Technique for K-Nearest Neighbor Search in Moving Objects Databases. In GeoInformatica 7(3), 2003, 255-273.

[16] P. Sistla, O. Wolfson, S. Chamberlain, S. Dao. Modeling and Querying Moving Objects. In ICDE 1997, 422432.

[17] J. Su, H. Xu, O. Ibarra. Moving Objects: Logical Relationships and Queries. In SSTD 2001, 3-19.

[18] Y. Tao, C. Faloutsos, D. Papadias, B. Liu. Prediction and Indexing of Moving Objects with Unknown Motion Patterns. In SIGMOD, 2004, 611-622.

[19] Y. Tao, D. Papadias, J. Zhai, Q. Li. Venn Sampling: A Novel Prediction Technique for Moving Objects. In ICDE, 2005, 680-691.

[20] M. Vazirgiannis, O. Wolfson. A Spatiotemporal Model and Language for Moving Objects on Road Networks. In SSTD, 2001, 20-35.

[21] O. Wolfson, B. Xu, S. Chamberlain, L. Jiang. Moving Object Databases: Issues and Solutions. In SSDBM 1998, 111-122. 